\title{
INCLUSION THEOREMS FOR GENERALIZED HAUSDORFF SUMMABILITY METHODS
}

\author{
LYNN C. KURTZ
}

The purpose of this note is to demonstrate how some of the classical Hausdorff inclusion theorems extend to the case where the sequences have their domains and ranges in topological vector spaces. We follow the terminology in [3] and [1] for the topological vector spaces and Hausdorff summability methods respectively.

Suppose $\left(X, T_{1}\right)$ and $\left(Y, T_{2}\right)$ are locally convex separated topological vector spaces. We denote by $L(X, Y)$ the linear functions from $X$ to $Y$ which are continuous with respect to the topologies $T_{1}$ and $T_{2}$.

Definition 1. If $f_{m n} \in L(X, Y)(m, n=0,1,2, \cdots)$, then the matrix $M=\left(f_{m n}\right)$ is called a summability method from $X$ to $Y$.

Suppose now that $\left(Z, T_{3}\right)$ is also a locally convex separated topological vector space.

Definition 2. If $M_{1}$ is a summability method from $X$ to $Y$ and $M_{2}$ is a summability method from $X$ to $Z$ with the property that for each sequence $\left\{x_{n}\right\}$ of points in $X$ for which $\left\{y_{m}\right\}=M_{1}\left(\left\{x_{n}\right\}\right)$ is $T_{2}$-convergent, the sequence $\left\{z_{m}\right\}=M_{2}\left(\left\{x_{n}\right\}\right)$ is $T_{3}$-convergent, then we say $M_{2}$ includes $M_{1}$. We indicate this by $M_{2} \supseteq M_{1}$.

We wish to consider Hausdorff methods $H(\mu)=\delta \mu \delta$, where $\mu$ $=\operatorname{diag}\left(\mu_{0}, \mu_{1}, \cdots\right), \delta$ is the differencing matrix, and $\mu_{i} \in L(X, Y)$ (for example). We denote by $N\left(\mu_{i}\right)$ the null space of $\mu_{i}$. Suppose we have two such Hausdorff methods $H_{i}=H_{i}(\mu)=\delta \mu_{i} \delta$, where $\mu_{i}$ $=\operatorname{diag}\left(\mu_{i 0}, \mu_{i 1}, \mu_{i 2}, \cdots\right)(i=1,2), \mu_{1 k} \in L(X, Y)$ and $\mu_{2 k} \in L(X, Z)$.

THEOREM 1. If $H_{2} \supseteq H_{1}$, then $N\left(\mu_{1 k}\right) \subseteq N\left(\mu_{2 k}\right)$ for $k=1,2, \cdots$.

Proof. Pick $x \in X$; then for a given $k$ define $\left\{\xi_{n}\right\}$ by $\xi_{n}=\theta$ if $0 \leqq n<k$ and $\xi_{n}=(n ! /(n-k) !) x$ if $k \leqq n$, and consider $\left\{\eta_{i m}\right\}=H_{i}\left(\left\{\xi_{n}\right\}\right)$ $(i=1,2)$. If $m \geqq k$, then

$$
\begin{aligned}
\eta_{i m} & =\sum_{n=0}^{m}\left(\begin{array}{c}
m \\
n
\end{array}\right) \Delta^{m-n} \mu_{i n} \cdot \xi_{n}=\sum_{n=k}^{m}\left(\begin{array}{c}
m \\
n
\end{array}\right) \Delta^{m-n} \mu_{i n} \cdot \frac{n !}{(n-k) !} x ; \\
& =\frac{m !}{(m-k) !} \sum_{n=k}^{m}\left(\begin{array}{c}
m-k \\
n-k
\end{array}\right) \Delta^{m-n} \mu_{i n} \cdot x \quad(i=1,2) ; \\
& =\frac{m !}{(m-k) !} \sum_{\nu=0}^{m-k}\left(\begin{array}{c}
m-k \\
\nu
\end{array}\right) \Delta^{m-k-\nu} \mu_{i, k+\nu} \cdot x=\frac{m !}{(m-k) !} \mu_{i k}(x) .
\end{aligned}
$$

Received by the editors October 13, 1967. 
Now, if $N\left(\mu_{1 k}\right)=\{\theta\}$, then $N\left(\mu_{2 k}\right) \supset N\left(\mu_{1 k}\right)$; if not, then we pick $x \in N\left(\mu_{1 k}\right)$ and $x \neq \theta$. For this $x, \eta_{1 m} \rightarrow \theta$ in the $T_{2}$ topology as $m \rightarrow \infty$. Since $H_{2} \supseteq H_{1}, H_{2}\left(\left\{\xi_{n}\right\}\right)$ must be $T_{3}$-convergent. If $k \geqq 1$, this implies $x \in N\left(\mu_{2 k}\right)$, for otherwise $\mu_{2 k}(x) \neq \theta$ and $\left\{(m ! /(m-k) !) \mu_{2 k}(x)\right\}$ cannot converge in the separated $T_{3}$ topology since $m ! /(m-k) ! \rightarrow \infty$ and the operation of scalar multiplication is continuous.

Definition 3. A summability method $M$ from $X$ to $Y$ is convergence preserving provided that if $\left\{x_{n}\right\}$ is $T_{1}$-convergent, then $M\left(\left\{x_{n}\right\}\right)$ is $T_{2}$-convergent.

THEOREM 2. If $\left(X, T_{1}\right)$ is fully complete, $\left(Y, T_{2}\right)$ is barrelled, and $\left(Z, T_{3}\right)$ is locally convex, and if $\mu_{1 k} \in L(X, Y)$ such that $\operatorname{dom} \mu_{1 k}=X$, range $\mu_{1 k}=Y(k=0,1,2, \cdots)$, and $\mu_{10}$ is $1-1$, and if $H_{2}$ is a Hausdorff method from $X$ to $Z$ such that $H_{2} \supseteq H_{1}$, then there exists a convergence preserving Hausdorff method $H(\phi)$ from $Y$ to $Z$ such that $H_{2}=H(\phi) H_{1}$.

Proof. By the statement $H_{2}=H(\phi) H_{1}$, we mean that $H_{2}\left(\left\{x_{n}\right\}\right)$ $=H(\phi)\left[H_{1}\left(\left\{x_{n}\right\}\right)\right]$. Let $\phi=\operatorname{diag}\left(\phi_{0}, \phi_{1}, \cdots\right)$, where $\phi_{k}\left[\mu_{1 k}(x)\right]$ $=\mu_{2 k}(x)$. If $\mu_{1 k}(x)=\mu_{1 k}\left(x^{\prime}\right)$, then $x-x^{\prime} \in N\left(\mu_{1 k}\right) \subset N\left(\mu_{2 k}\right)$, since $\mu_{10}$ is 1-1 and $H_{2} \supseteq H_{1}$. Hence $\mu_{2 k}(x)=\mu_{2 k}\left(x^{\prime}\right)$ and $\phi_{k}$ is well defined; it is clear that $\phi_{k}$ is linear.

Since $\mu_{1 k}$ is continuous and $X$ is fully complete, it follows that $N\left(\mu_{1 k}\right)$ is closed and hence $X / N\left(\mu_{1 k}\right)$ is fully complete [2, p. 114]. If we define $\bar{\mu}_{1 k}$ from $X / N\left(\mu_{1 k}\right)$ to $Y$ by $\bar{\mu}_{1 k}(\bar{x})=\mu_{1 k}(x)(x \in \bar{x})$, then $\bar{\mu}_{1 k}$ is 1-1 and continuous (in the quotient topology) [2, p. 78] onto $Y$ and hence is an isomorphism $\left[2\right.$, p. 116]. We can now see that $\phi_{k}$ is continuous, for suppose $V$ is an open set in $Z$. Then $U=\mu_{2 k}^{-1}(V)$ is open in $X$. If $\nu_{k}$ is the canonical map of $X$ to $X / N\left(\mu_{1 k}\right)$ given by $\nu_{k}(x)=\bar{x}$, then $\bar{U}=\nu_{k}(U)$ is open in the topology of $X / N\left(\mu_{1 k}\right)$ and $W=\bar{\mu}_{1 k}(\bar{U})$ is open in $Y$, since $\bar{\mu}_{1 k}$ is an isomorphism. But $W=\phi_{k}^{-1}(V)$, so $\phi_{k}$ is continuous; and we have shown $\phi_{k} \in L(Y, Z)$. The argument used here generalizes a result of Sard [4].

It remains to be shown that $H_{2}=H(\phi) H_{1}$ and that $H(\phi)$ is convergence preserving. The first is easy:

$$
H(\phi) H_{1}=(\delta \phi \delta)\left(\delta \mu_{1} \delta\right)=\delta\left(\phi \mu_{1}\right) \delta=\delta \mu_{2} \delta=H_{2} .
$$

Suppose $\left\{\xi_{n}\right\}$ is convergent in $\left(Y, T_{2}\right)$, and consider the set of equations

$$
\xi_{m}=\sum_{n=0}^{m}\left(\begin{array}{c}
m \\
n
\end{array}\right) \Delta^{m-n} \mu_{1 n} \cdot x_{n} \quad(m=0,1, \cdots) .
$$

This is a triangular system, and since $\mu_{1 n}$ is onto $Y$, we may find a 
sequence (in fact many sequences) $\left\{x_{n}\right\}$ which satisfies it. For any such $\left\{x_{n}\right\}$, the sequence $\left\{\eta_{m}\right\}$ given by $\left\{\eta_{m}\right\}=H_{2}\left(\left\{x_{n}\right\}\right)$ converges in $\left(Z, T_{8}\right)$, since $H_{2}$ is convergence preserving. But $H_{2}\left(\left\{x_{n}\right\}\right)$ $=H(\phi)\left[H_{1}\left(\left\{x_{n}\right\}\right)\right]=H(\phi)\left(\left\{\xi_{n}\right\}\right)$. Hence $H(\phi)$ is convergence preserving.

\section{REFERENCES}

1. G. H. Hardy, Divergent series, Clarendon Press, Oxford, 1949.

2. L. C. Kurtz and D. H. Tucker, Vector-valued summability methods on a linear normed space, Proc. Amer. Math. Soc. 16 (1965), 419-428.

3. A. P. and W. J. Robertson, Topological vector spaces, Cambridge Univ. Press, Cambridge, 1964.

4. Arthur Sard, Integral representations of remainders, Duke Math. J. 15 (1948), 333-345.

Arizona State University 